\title{
The Effect of Different Ratios of Malonic Acid to Plyvinylalcohol on Electrochemical and Mechanical Properties of Polyacrylonitrile Electrospun Separators in Lithium-Ion Batteries
}

\author{
Fartash Khodaverdi ${ }^{1}$, Mehran Javanbakht ${ }^{2}$, Ali Vazirii" ${ }^{1 *}$ Mehdi Jahanfar ${ }^{3 *}$ \\ 1 Department of Petroleum and Chemical Engineering, Science and Research Branch, Islamic Azad University, 11369 Tehran, \\ P. O. B. 1477893855 , Iran \\ 2 Department of Chemistry, Amirkabir University of Technology, 11369 Tehran, P. O. B. 1591634311, Iran \\ ${ }^{3}$ Department of Biological Engineering and Biotechnology, Shahid Beheshti University, 11369 Tehran, P. O. B. 1983969411, Iran \\ *Corresponding authors, e-mail: a.vaziri@srbiau.ac.ir; jahanfar61@gmail.com
}

Received: 22 January 2021, Accepted: 18 February 2021, Published online: 21 June 2021

\begin{abstract}
The present study aimed to investigate the mechanical, thermal, and electrochemical properties of Polyacrylonitrile (PAN) electrospun separators in the presence of Polyvinylalcohol (PVA) hydrophilic materials and Malonic Acid (MA) crosslinker inside the lithium-ion batteries. The results showed that the M3 modified separator with the MA to PVA+MA (wt./wt.) optimum ratio of $37.5 \%$ had the best performance in all tests. This separator had a value of $3.16 \mathrm{mS} / \mathrm{cm}$ in the ion conductivity test. Additionally, it had an electrolyte uptake of $1172 \%$ ( 2.39 times more than the neat PAN separator) and thermal shrinkage of $7.4 \%$ at $180{ }^{\circ} \mathrm{C}$, where this value was $14.5 \%$ for neat PAN separator at the same experimental condition. Furthermore, the acceptable performance in the battery performance tests was compared with other separators.
\end{abstract}

Keywords

separator, lithium-ion batteries, electrolyte uptake, MA to PVA ratio

\section{Introduction}

Considerable research attention has been paid to the fundamental need for clean energy and novel approaches for decreasing the emission of deleterious greenhouse gasses $[1,2]$. The performance/behavior optimization of these types of power supplies has been extensively investigated due to the upward progress in the technology of second-generation (rechargeable) battery systems [3]. The lithium-ion battery is one of the most applicable types of batteries with high energy density, significant flexibility, and low weight [3]. Lithium-ion batteries consist of several separators considered as porous membranes whose operational performance through the lithium-ion battery is entirely different from their performance in the membrane-based gas/liquid separation. These separators prevent anode and cathode contact and short circuiting. In addition, they play an important role in improving the performance of lithium-ion batteries $[4,5]$.
The electrospinning process, as an appropriate technique to manufacture submicron-sized polymeric fibers, has numerous important operational capabilities such as the simplicity of production parameter and suitable morphology control [6]. Electrospun fibers are generally collected in the nonwoven form and extensively used as semi-permeable separators, filters, and optical/electronic devices [7]. Given the proper performance of non-woven separators and the importance of cost-effectiveness, the electrospinning process can be considered as a suitable procedure for preparing separators in lithium-ion batteries [8]. Huai et al. [9] prepared a structure-specific PAN membrane to be applied as a separator in lithium-ion batteries and found that the structure-specific separator made of PAN was very promising for use in high safety lithium-ion batteries [9]. In another study, Zhu et al. [10] investigated PAN/PVDF separators to find the best separator with suitable mechanical, thermal, and dimensional resistance in lithium-ion batteries. 
PAN and PVDF with different ratios were used as polymer, and dimethylformamide (DMF) was applied as a solvent for separator fabrication. The SEM test was performed to examine the morphology of the separators. The findings indicated that the sample with the same percentage of PAN and PVDF had the best performance in its tensile strength test. The sample made of only PAN polymer demonstrated the best performance in terms of ion conductivity, electrolyte uptake, thermal stability, and charge/discharge process [10]. In addition, Agubra et al. [11] used $12 \mathrm{wt} \%$ of commercial PAN with a molecular weight of 150,000 in DMF solvent at $60^{\circ} \mathrm{C}$ for the industrial production of PAN separators via the force spinning technique. SEM and DSC tests were applied to evaluate the separators. The results indicated increments in the uptake rate (from 82 to $328 \%$ ) and ion conductivity (from 0.33 to $1.23 \mathrm{mS} / \mathrm{cm}$ ) in comparison to Commercial Polypropylene (PP) separator. The force spinning was introduced as an effective technique to produce high quality, industry-leading PAN nanofibers with high ion conductivity, low surface interaction, and low electrochemical oxidation [11]. He et al. [12] examined PAN/ PVA separators to prepare lithium battery separators. PVA/ PAN in the mixed matrix membranes with weight ratios of 0:100,10:90, 20:80, and 40:60 (wt./wt.) were carefully evaluated and denoted as BM-0, BM-1, BM-2, and BM-3, respectively. The results showed that mixed matrix membranes with weight ratios of PVA/PAN $=10: 90$ and 20:80 had a substantial effect on the morphology of both top and bottom surfaces. This important property highlighted the high electrolyte uptake (up to $585 \%$ ), brilliant thermal stability, appropriate ionic conductivity, and good electrochemical stability (up to $5.28 \mathrm{~V}$ ). The exothermic peaks associated with decomposition centered at 324, 302, 299, and $291^{\circ} \mathrm{C}$ were seen in BM-0, BM-1, BM-2, and BM-3, respectively. The liquid uptake was $68,369,505,510$, and $585 \%$ for Celgard 2320, BM-0, BM-1, BM-2, and BM-3, respectively. For the Celgard 2320 separator, an identifiable anodic peak onset was observed at $4.24 \mathrm{~V}$ due to its association with the decomposition of the carbonate solvent. Insertion of PVA into PAN polymer matrix resulted in the higher decomposition voltage of mixed membranes with $4.72,4.62,5.05$, and $5.28 \mathrm{~V}$ in comparison to the application of Celgard 2320 separator. After 200 cycles, the capacity retention of the cells for Celgard 2320, BM-0, BM-1, BM-2, and BM-3 were 58, 1, 94, 96, and $40 \%$, respectively. The cell, which was assembled via BM-1 and BM-2 membranes, demonstrated a better cycle performance as compared with the one assembled with Celgard 2320. Therefore, the abovementioned advantages makes this separator a suitable option for application in lithium metal batteries [12].

Gaaz et al. proved that PVA could become a structurally different polymer with proper mechanical properties and thermal stability due to the thermal stability of HNTs although it had low strength and thermal stability for some applications, [13]. Crosslinking is considered as a well-known technique which is generally applied to enhance the mechanical/thermal properties and increase the solubility of polymers. Apart from the PVA, glyoxal glutaraldehyde (GA) is a widely used crosslinker for polymers which primarily includes amine groups such as proteins $[14,15]$. Other studies showed that the crosslinking process of PVA could be performed by applying dicarboxylic acids like malonic acid (MA) with sulphuric acid as a catalyst to obtain esterification $[16,17]$. The use of MA crosslinked HNTs-PVA composites significantly improved the thermal/mechanical properties [13].

Ivanishchev et.al in their own series of studies investigating the mechanism of lithium ion transfer in the battery and the parameters affecting it provided a model. Using this model, a suitable prediction for the voltammetry cycle was made. Their model was presented based on the structural features, the synthesis method, the relationships between the synthesis conditions, the structural and electrochemical characteristics of materials. Taking account of the peculiarity of this model the diffusion parameters of lithium transport to be correctly determined. They also modeled impedance spectra using electrical equivalent circuit (EEC) [18-22]. Bolto et al. examined the crosslinking of PVA with the dicarboxylic acid oxalic, malonic, and citric acids with sulphuric acid as a catalyst at $90-120^{\circ} \mathrm{C}$ [16]. Malic acid $\left(\mathrm{HO}_{2} \mathrm{C}-\mathrm{CH}_{2}-\mathrm{CH}(\mathrm{OH})-\mathrm{CO}_{2} \mathrm{H}\right)$ had a more effective crosslinking agent in comparison to GA or other aldehydes and acids in the fabrication of thin-film composite membranes made by coating a polysulphone UF membrane with PVA [23]. In another study, Qiu et al. [24]. Studied the polyvinyl alcohol (PVA) as a thermoplastic and biocompatible petroleum-based polymer. PVA is a rare polymer with a carbon-carbon single bond backbone which is entirely biodegradable. In addition, it is considerably hydrophilic and soluble in water due to the existence of hydroxyl $(-\mathrm{OH})$ groups on alternating carbon atoms, which helps improve its degradation through hydrolysis [25]. Crosslinking has been widely applied to modify the mechanical and thermal properties as well as the decline solubility of many polymers [14, 15]. When crosslinking is applied as resin, the crosslinked polymers can improve the composite 
properties. Crosslinking is a chemical process in which two or more molecules are joined at disparate locations along their length by covalent bonds. Glutaraldehyde and glyoxal are two commonly applied crosslinkers for polymers consisting of amine groups such as proteins [14, 15]. The two aforementioned crosslinkers are relatively toxic although they are efficacious in crosslinking polymers. Therefore, less toxic crosslinkers are preferred in the crosslinking reaction for preserving the biodegradability of the polymers. Malonic Acid (MA) is a dicarboxylic acid, made of chloroacetic acid, could be used as a crosslinker. Regarding toxicity, it is a better option as a crosslinker in comparison to glutaraldehyde or glyoxal. Some investigators have examined the crosslinking process of PVA using dicarboxylic acids, including MA, with sulfuric acid as a catalyst to obtain esterification $[16,17]$. However, the efficiency of crosslinking has not been very satisfactory. More importantly, previous studies failed to take into account the mechanical/thermal properties of PVA after crosslinking. Swelling analyses were implemented for non-crosslinked (control) and crosslinked PVA as well as for the resin in the crosslinked HNT-PVA nanocomposites to assess the level of crosslinking. The results indicated that the percentages of the crosslinked PVA and the resin in the crosslinked HNT-PVA nanocomposites were 93.1 and $94.8 \%$, respectively, while the control PVA was completely dissolved during the sol-gel test. Based on the high gel percentage, the PVA was crosslinked by MA when it was catalyzed by phosphoric acid. In addition, the swelling powers of the crosslinked PVA and the resin in the crosslinked HNT-PVA nanocomposites were 52.3 and $51.0 \%$, respectively, which were much lower than the control (non-crosslinked) PVA (107.3\%) [25]. The present study mainly aims to examine the effect of using MA and PVA on the momentous properties of PAN electrospun separators. Additionally, this study investigates the impact of the simultaneous alteration of the MA to PVA ratios to introduce the best MA to PVA ratio for increasing the electrochemical, morphological, and mechanical properties of fabricated PAN/PVA/MA electrospun separators in the lithium-ion batteries.

\section{Experimental study}

\subsection{Preparation of electrode and cell assembly}

A uniform and monotonous mixture of 80 wt.\% $\mathrm{LiFePO}_{4}$ (EnnoPro Group, Shenzhen, Guangdong, China) with 10 wt.\% carbon black (Printex XE, Evonik, Germany) and 10 wt.\% PVDF (Solvay, Solef ${ }^{\circledR}$ 5130) was prepared by utilizing N-methyl-2-pyrrolidone (NMP, Merck, Darmstadt,
Germany) as an applicable chemical dispersant. Then, to prepare the cathode electrodes, the slurry mixture was coated onto an aluminum foil, dried, and pressed [26]. The lithium-ion half cell (2032-type coin) was precisely prepared in a glove box filled with argon by locating the separator soaked in a liquid electrolyte (Dimethyl carbonate (DMC)/Ethylene carbonate (EC)/Diethyl carbonate (DEC) $(\mathrm{DMC} / \mathrm{EC} / \mathrm{DEC}=1 / 1 / 1$ by volume $)$ with $1.0 \mathrm{M}$ lithium hexafluorophosphate (LiPF6) as a chemical electrolyte) between the lithium metal anode and the cathode [3].

\subsection{Preparation of the non-woven separator}

PVA, MA, and PAN were dissolved in DMF to prepare the PAN/PVA/MA solutions by considering various blend ratios. Next, PAN/PVA/MA non-woven separator was manufactured by the electrospinning method. Table 1 shows the detailed information about the required percentages of PVA, MA, and PAN for preparing different separators.

The single nozzle electrospinning setup (HV35P OV, Fanavaran Nano Meghias, Iran) was applied in this study. The experiments lasted for about 12 hour under the ambient conditions. The temperature and relative humidity were fixed at $25 \pm 3{ }^{\circ} \mathrm{C}$ and $45 \pm 5 \%$, respectively. The flow rate and high voltage required for making the polymer solutions electrospun were $1.5 \mathrm{~mL} . \mathrm{h}^{-1}$ and $20 \mathrm{kV}$, respectively. The distance between the collector and the needle tip was $100 \mathrm{~mm}$.

\subsection{Characterization}

\subsubsection{FTIR analysis}

The FTIR spectrophotometer (NEXUS-870, Thermo Nicolet Corporation, Waltham, MD) was applied to evaluate the separator FTIR spectra in the wavenumber range of 400 $4000 \mathrm{~cm}^{-1}$ at room temperature with the resolution of $1 \mathrm{~cm}^{-1}$.

\subsubsection{Morphology}

Scanning Electron Microscopy (SEM, Hitachi SU3500, Japan) was utilized to investigate the morphology of the separators. Atomic Force Microscopy (AFM) was performed via Ara-A.F.M scanning probe optical microscope

Table 1 Names and weight percentages of PAN, PVA, and MA

\begin{tabular}{lccc}
\hline $\begin{array}{l}\text { Separators } \\
\text { Name }\end{array}$ & $\begin{array}{c}\text { PAN } \\
(\text { wt.\%) }\end{array}$ & $\begin{array}{c}\text { PVA and MA } \\
(\text { wt. \%) }\end{array}$ & $\begin{array}{c}\text { MA (wt.) } \\
\text { PVA and MA (wt.) } \times 100\end{array}$ \\
\hline PAN & 15 & 0 & - \\
M1 & 15 & 5 & 12.5 \\
M2 & 15 & 5 & 25.0 \\
M3 & 15 & 5 & 37.5 \\
M4 & 15 & 5 & 50.0 \\
\hline
\end{tabular}


(ARA model 0101/A, Iran) to evaluate the surface roughness of the separators. In addition, the surface roughness was predicted based on the height profile of the AFM images in the IMAGER J software [27].

\subsubsection{Calculation of the electrolyte uptake}

The desirable time spent for the immersion process of microporous separators in the liquid electrolyte at room temperature was approximately $60 \mathrm{~min}$. Then, the excessive electrolytes were properly removed. The evaluation trend of separator weights takes place both before and after the immersion process in the liquid electrolyte. Eq. (1) was used to calculate the electrolyte uptake [26]:

Electrolyte Uptake $(\%)=\frac{\left(w-w_{0}\right)}{w_{0}} \times 100$,

where $w$ and $w_{0}$ represent the weights of the separators after and before the immersion process in the liquid electrolyte, respectively.

\subsubsection{Measurement of wettability}

A contact angle goniometer was used to measure the surface wettability and hydrophilicity of the separators. For this purpose, a commercial drop shape analysis system (Kruss GmbH, Hamburg, Germany) was operationally applied to measure the static contact angle of the separators with deionized water [28].

\subsubsection{Evaluation of tensile}

Instron 5566 (Instron Co., U.S.A) is an appropriate apparatus applied for measuring the tensile strength of the separators at room temperature. The test rate and the sample size were $5 \mathrm{~mm} / \mathrm{min}$ and $100 \mathrm{~mm} \times 10 \mathrm{~mm}$ for separators, respectively.

\subsubsection{Measurement of thermal shrinkage}

To measure the dimensional stability of the separators at high temperatures, their thermal shrinkage was calculated using Eq. (2) [29]:

Thermal shrinkage $(\%)=\frac{\left(S-S_{0}\right)}{S} \times 100$,

where $S$ and $S_{0}$ are the separator areas before and after the heat treatment, respectively. The separators were placed in an oven at $140-180{ }^{\circ} \mathrm{C}$ for approximately $30 \mathrm{~min}$.

\subsubsection{Evaluation of ion conductivity}

The bulk resistance of the prepared liquid electrolyte-soaked separators located between two stainless steel plate electrodes was measured using Electrochemical Impedance Spectroscopy (EIS) at room temperature and the voltage amplitude and frequency range of $10 \mathrm{mV}$ and $10^{-1}-10^{5} \mathrm{~Hz}$, respectively. Furthermore, ionic conductivity was measured using the PGSTAT302N Galvanostat/ Potentiostat Auto lab. Eq. (3) was applied to calculate the ion conductivity [30]:

$\sigma=\frac{d}{A \times R_{b}} \times 100$,

where $\sigma, d, A$, and $R_{b}$ denote the ion conductivity, the thickness of the separator, the effective area, and the bulk resistance of the separator, respectively. The thickness of the separators was measured using a digital micrometer (Mitutoyo Corp, Japan) with an accuracy of $1 \mu \mathrm{m}$.

\subsubsection{Measurement of battery performance}

The NEWARE battery testing system with the operating voltage range of $2.5-4.2 \mathrm{~V}$ was applied to perform the discharge rate tests at the $\mathrm{C}$-rates of $0.1 \mathrm{C}, 0.2 \mathrm{C}, 0.5 \mathrm{C}, 1.0 \mathrm{C}$, $2.0 \mathrm{C}$, and $0.1 \mathrm{C}$. The cycling performance of the desired fabricated cells by applying disparate separators was evaluated at $0.5 \mathrm{C}$ for 100 . For activation, all fabricated cells were cycled twice in the voltage range of 2.5 to $4.2 \mathrm{~V}$ at a current rate of $0.1 \mathrm{C}$.

\section{Results and discussion}

\subsection{FTIR spectroscopic analysis}

Fig. 1 shows the FTIR spectra of PAN, M1, M2, M3, and M4 separators fabricated with PAN/PVA/MA polymer composites. As expected, when the MA was prepared to react with PAN/PVA, the carboxylic groups $(\mathrm{COOH})$ in MA react with the hydroxyl groups $(\mathrm{OH})$ in the PVA forming ester linkages. The broadband at $3500-3600 \mathrm{~cm}^{-1}$ wavenumber as a result of the $\mathrm{O}-\mathrm{H}$ stretching vibration caused by the strong intra-molecular and intermolecular

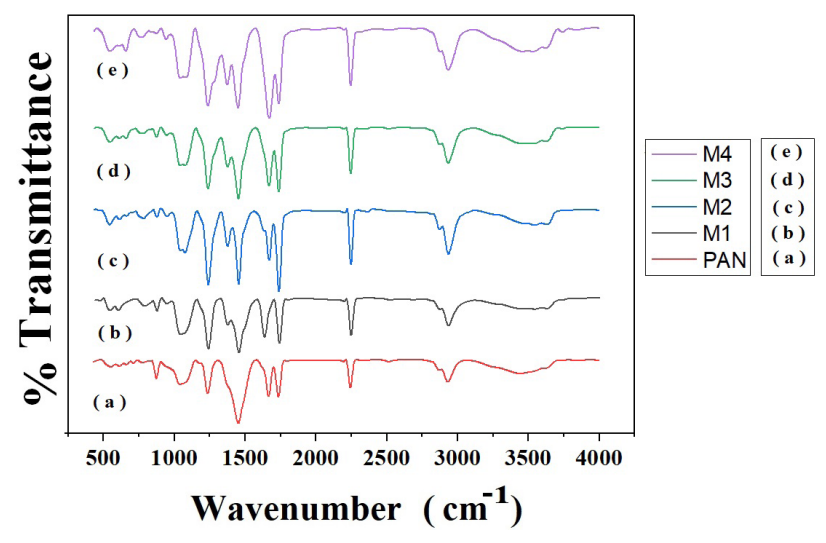

Fig. 1 The FTIR spectra of the separators 
hydrogen bonding [31]. The absorbance intensity ratio for $\mathrm{O}-\mathrm{H}$ to $\mathrm{C}-\mathrm{H}$ bands indicated a decrease for control PAN in comparison to the crosslinked PAN/PVA/MA, which shows a deterioration in the $\mathrm{O}-\mathrm{H}$ groups and proves the crosslinking of PAN/PVA by MA [32]. Absorptions at the wave numbers of 2800-2900 $\mathrm{cm}^{-1}$ are confirmed because of the $\mathrm{CH}$ asymmetric and symmetric stretching [33]. Additionally, it is related to the stretching of aliphatic $\mathrm{C}-\mathrm{H}$ bonds [32]. This lower absorption indicated a decrease in the O-H groups and confirmed the crosslinking of PVA and PAN by MA, which is in line with the results of previous research $[13,32,34]$. For all prepared separators, the absorption peak at the wavenumber of $2225 \mathrm{~cm}^{-1}$ is associated with the stretching vibration of nitrile groups. The sharp absorption at $1650-1750 \mathrm{~cm}^{-1}$ (stretching of $\mathrm{C}=\mathrm{O}$ ) for non-crosslinked specimen was weak (intensity ratio of $\mathrm{C}=\mathrm{O}$ to $\mathrm{C}-\mathrm{H}$ ), which indicates the presence of carbonyl $(\mathrm{C}=\mathrm{O})$ in the nanocomposites from the no hydrolyzed acetate group [32, 34]. In other words, in addition to the no hydrolyzed vinyl acetate groups, some residual unreacted carboxylic groups from MA and carboxylic ester groups (Fig. 1) and crosslinked PAN/PVA contributed to the stronger $\mathrm{C}=\mathrm{O}$ absorption [25].

A sharp peak can be easily seen in the wave number range of $1100 \mathrm{~cm}^{-1}$, which could be due to the stretching $\mathrm{O}-\mathrm{H}$ group and the reaction of PAN and PVA with MA which eventuated in a significant deterioration in the intensity of O-H group. The peaks at $500-550 \mathrm{~cm}^{-1}$ is related to $\mathrm{CH} 2$ [35]. It could be speculated that the changes observed in the peaks trend were mostly related to the influence of PVA and MA on the combined polymer.

\subsection{Morphology}

The electrospun and non-woven membranes were randomly overlaid layer-by-layer, and no beads existed microscopically in the fibers. The presence of beaded fibers is an important problem in the electrospun Nano fibrous membrane separator, which can negatively affect the lucrative properties of the electrospun Nano fibrous membranes [36]. Fig. 2 shows the surface SEM images of PAN, M1, M2, M3, and M4 separators. As shown the bead free fibrous structure with large pores number is the principal structural characteristic of all the fabricated separators. Viscosity is a momentous parameter which plays a key role in changing the fiber size [37]. The diameter of the average fibers was controlled by regulating the concentrations of polymers and electrospinning parameters [36]. Enhancement in the amount of PVA and MA polar materials in the PAN solution resulted in substantial improvement in the viscosity of the solution and consequently the diameter of the fibers [38, 39]. The forces caused by the entanglement of the polymer chains entering the jet under the electric field increased by increasing the polarity of the solution and the amount of charge carried by jet, which resulted in fabricating smaller fiber diameters [40]. A decrement in die swell phenomenon was seen in crosslinked membranes, which can facilitate the production of fibers with smaller diameters [20, 40].

Fig. 2 shows the high magnification of SEM image for the crosslinked membranes in which the bonding structures were formed among the adjacent fibers of PAN/PVA [41]. This change and creation of the network in Fig. 2 are related to visible M1 to M4 samples.

\subsection{Thermal shrinkage}

The thermal shrinkage of separators occurs when the temperature increases to the softening temperature [5]. Thermal shrinkage of the separator takes pace due to the density gradient between the crystalline and the amorphous polymer phases [42]. The shrinkage amount can be attributed to mechanical and thermal resistance $[25,43]$.

Table 2 shows the thermal shrinkages for PAN, M1, M2, M3, and M4 separators at temperatures of 140, 160 and $180^{\circ} \mathrm{C}$. It is worth noting that PVA has lower melting point and lower thermal resistance compared to neat PAN at high
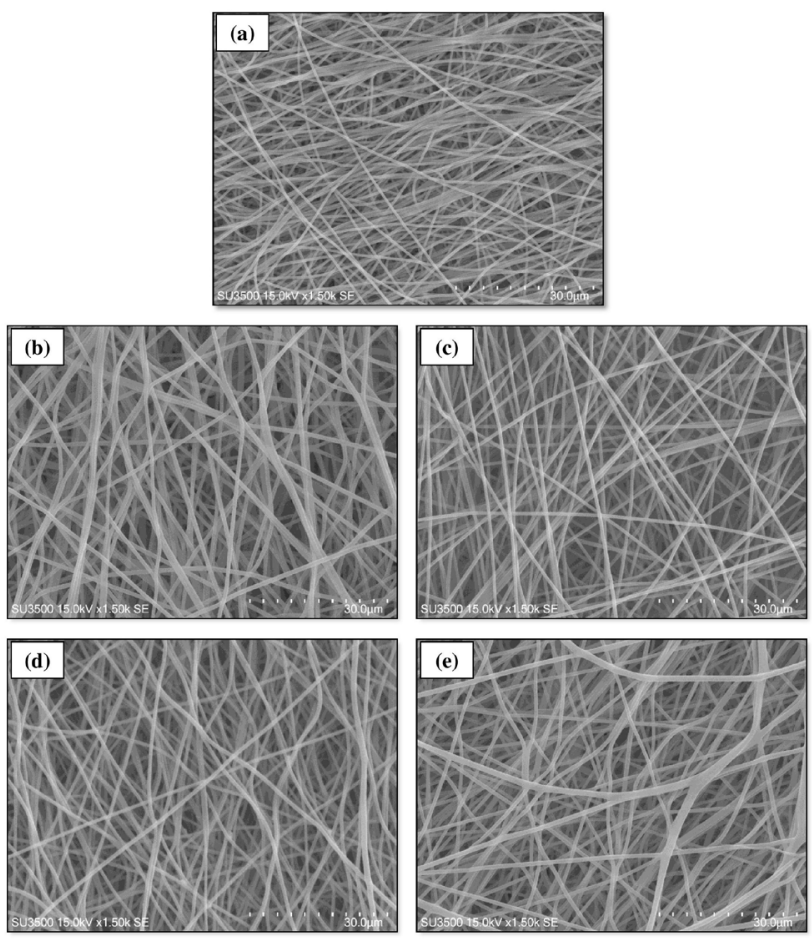

Fig. 2 SEM images of (a) PAN, (b) M1, (c) M2, (d) M3, and (e) M4 separators 
Table 2 Thermal shrinkages and tensile strengths of PAN, M1, M2, M3, and M4 separators

\begin{tabular}{|c|c|c|c|c|c|c|}
\hline Separator & $\begin{array}{l}\text { Average fiber } \\
\text { diameter }(\mu \mathrm{m})\end{array}$ & $\begin{array}{c}\text { Thermal shrinkage } \\
140{ }^{\circ} \mathrm{C}(\%)\end{array}$ & $\begin{array}{c}\text { Thermal shrinkage } \\
160^{\circ} \mathrm{C}(\%)\end{array}$ & $\begin{array}{c}\text { Thermal shrinkage } \\
180^{\circ} \mathrm{C}(\%)\end{array}$ & $\begin{array}{l}\text { Tensile Strength } \\
\text { (MPa) }\end{array}$ & Contact Angle $\left({ }^{\circ}\right)$ \\
\hline PAN & 0.650 & 4.8 & 7.4 & 14.5 & 6.1 & 86 \\
\hline M1 & 0.940 & 5.3 & 7.8 & 16.2 & 5.6 & 76 \\
\hline M2 & 1.180 & 4.5 & 6.0 & 9.9 & 5.9 & 61 \\
\hline M3 & 1.370 & 2.9 & 4.8 & 7.4 & 7.1 & 36 \\
\hline M4 & 1.315 & 2.9 & 4.9 & 7.7 & 6.8 & 42 \\
\hline
\end{tabular}

temperatures [44]. However, as mentioned in SEM section, no shrinkage increment occurred, and there was some resistance to deformation at high temperatures in the M1, M2, M3, and M4 separators by the assistance of MA cross-linker and the network formation between the fibers. The amount of M3 thermal shrinkage at $180{ }^{\circ} \mathrm{C}$ is $7.4 \%$ while the PAN thermal shrinkage considering similar operational conditions is $14.5 \%$. The formation of the network in separator created a structure in which the polymer chains were interconnected molecularly, which prevented from moving the polymer chains rapidly and easily, and increased the resistance of the separator to further deformation due to an increase in the temperature [45]. An improvement in the shrinkage resistance rate occurred in separators by increasing the amount of MA crosslinker and the formation of a network structure (Fig. 3). However, the trend changed in M4 compared to $\mathrm{M} 3$ due to the saturation of $\mathrm{OH}$ functional groups in PVC by MA or the lack of its participation in the related reaction. As the MA further increases, it can play the role of a plasticizer in the mixture and reduce the shrinkage resistance due to its inherent properties. On the other hand, a decrement in the overall resistance of the M4 membrane can be observed compared to M3 by reducing the amount of PVA, which has higher thermal resistance than MA.

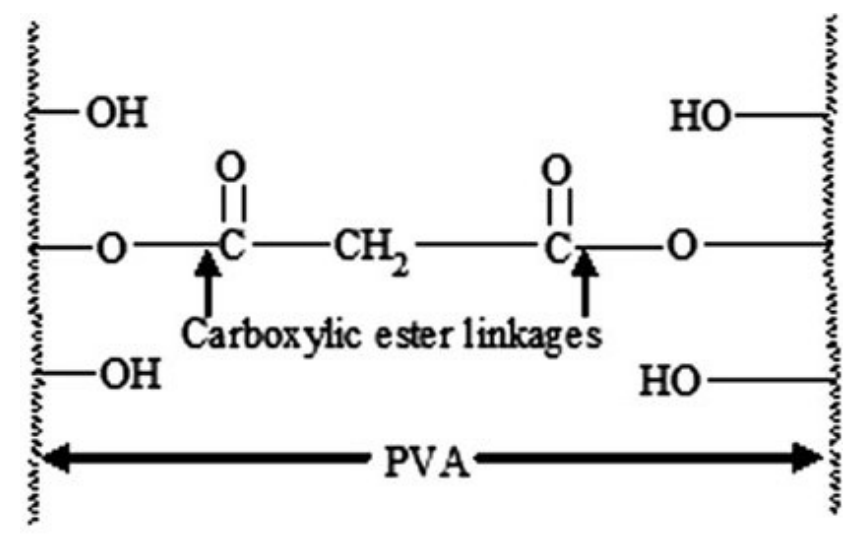

Fig. 3 The schematic view of PAN and PVA using MA

\subsection{Tensile strength}

Table 2 shows the results of tensile strength for PAN, M1, M2, M3, and M4 separators. Tensile strength is directly related to the length of the polymer chains in terms of their length and how they are bonded to each other [10]. In the prepared samples, a substantial decrement in tensile strength occurred [44] by increasing the amount of PVA hydrophilic material to neat PAN, which had less tensile strength in comparison to PAN [41]. The polymer network of PAN/PVA/MA could be tailored into a threedimensional network structure at the molecular level, which would considerably enhance the strength for the whole membrane [43]. Due to the interpenetrations of PAN/PVA/ MA fibers, the PVA would immobilize the PAN to some extent by forming connection points. Furthermore, the toughness of PAN membrane improved from $3.61 \mathrm{Jg}^{-1}$ to $6.23 \mathrm{Jg}^{-1}$ [32] because of adding PVA. In comparison with PVA fibers, the PAN fibers play a role in the decreased tensile strength [32, 34]. However, increasing the amount of MA acted as a crosslinking agent by decreasing the PVA effect, creating a chain link, and significantly enhancing the tensile strength in the samples (M3 = 7.1 MPa). Hence, the alterations can be evaluated now. The tensile strength amount reduced in the M1 sample, where the MA level in the combination between MA and PVA was low and more PVA value was used in the membrane composition compared to MA. In M2 and M3 samples by increasing the MA in the MA/ PVA combination, the increment in the tensile strength could be justified by enhancing the number of chains due to the greater presence of MA. As the amount of PVA in the samples increased, some samples could not participate in the reaction and act as plasticizers due to their intrinsic properties, which may be the reason for the reduction in tensile strength in the M4 sample. Further, plasticizing resulted in increasing elongation at break from $100 \%$ to $400 \%$ in comparison with plasticized starch while Young's modulus and tensile strength decreased by one order of magnitude [46]. 


\subsection{The wettability of the separators}

The wettability of the separators plays an essential role in the operational efficiency of the lithium-ion batteries. The surface hydrophilicity was investigated to analyze the wettability of the separators [47]. To this end, the water contact angle with the separators was measured [48]. The obtained contact angle PAN, M1, M2, M3, and M4 separators are presented in Table 2. The addition of PVA/MA led to a significant increment in the hydrophilicity of PAN separator and a substantial decrease in the water contact angle [49]. Several studies showed that the MA and PAN had significant polarities, which could influence the wettability, conductivity behavior, and ion transport [50]. The presence of $\mathrm{OH}$ polar groups in MA and PVA increased the possibility of polar-to-polar relation with a water molecule, which resulted in higher water absorption in M3 and M4 samples with a water contact angle of 360 and $42^{\circ}$, respectively [49]. An increase in the amount of MA eventuates in increasing the amount of the network structure in the separator. However, when the MA amount exceeded the saturation level, the MA acted as a plasticizer filler and considerably reduced the surface roughness [49]. The surface roughness of the separators with MA significantly increased in comparison to the PAN separator. Further, the neat PAN separator displayed Root Mean Squared (RMS) surface roughness value of $6 \mathrm{~nm}$ for M1: $5.2 \mathrm{~nm}, \mathrm{M} 2: 6.1 \mathrm{~nm}, \mathrm{M} 3: 8.4 \mathrm{~nm}$, and M4: $8.2 \mathrm{~nm}$. The surface roughness results could be justified by considering the fact that the surface roughness in M1 was lower than the PAN. The considerable decrease in the overall surface roughness is due to the lower amount of PVA surface roughness as compared to PAN [49]. By increasing MA into the solution as a cross-linking agent, the surface roughness increased due to the formation of network and tentacle on the surface. The results are consistent with Wenzel's theory, which improves hydrophilicity [49, 51]. Theories of Wenzel and Cassie-Baxter, which are used for porous surfaces, can be applied to describe the behavior of the droplets on surfaces. Since the surface of the prototype had a contact angle of $86^{\circ}$, increasing the roughness of the surface could increase the level of the surface hydrophilicity. This theory, along with the intrinsic properties of the substances added to the PAN, can justify the wettability behavior [52]. The pure PAN separator had the highest contact angle value of $86^{\circ}$. In comparison, the blend separators showed a lower contact angle, which indicated the improved hydrophilicity of the modified PAN separators by the addition of hydrophilic materials (PVA and MA). A similar improvement in the hydrophilicity of the separators was reported by Li et al. [49].

\subsection{Liquid electrolyte uptake}

Electrolyte uptake is an indispensable parameter to evaluate the electrolyte absorption capacity of the separators [3]. The separators applied in the lithium-ion batteries should be practically wetted with the electrolyte and have proper electrolyte uptake to increase their stability and simplify their assembly [53]. Fig. 4 shows the electrolyte uptakes of the PAN, M1, M2, M3, and M4 separators. As shown in Fig. 4, the electrolyte uptake of M3 separator is $1172 \%$. The porosity and wettability of the separators are the design/operational parameters, which directly influence the electrolyte uptake [54]. As shown in Table 2, the contact angle of M3 separator is significantly lower than the PAN separator, which could be attributed to higher electrolyte uptake and is in line with the findings of some previous studies [3, 26].

\subsection{Ion conductivity}

Figs. 5 and 6 shows the experimental results of the ion conductivity analysis for PAN, M1, M2, M3, and M4 separators. As shown in Fig. 5, an increase in the amount of liquid electrolyte in the pores of the separator and lithium-ions migration from the separators can positively enhance the ion conductivity by forming the separator network structure [55]. Since the electrolyte uptake of these separators

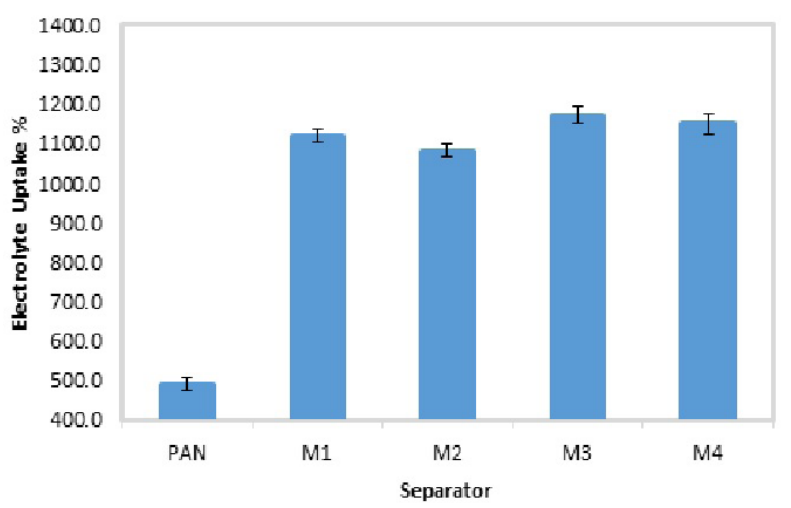

Fig. 4 The electrolyte uptakes of PAN, M1, M2, M3, and M4 separators

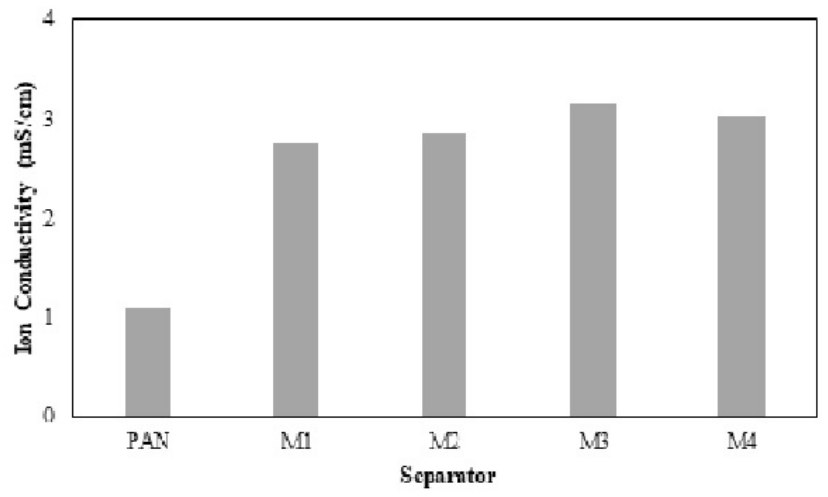

Fig. 5 Ion conductivity of PAN, M1, M2, M3, and M4 separators 


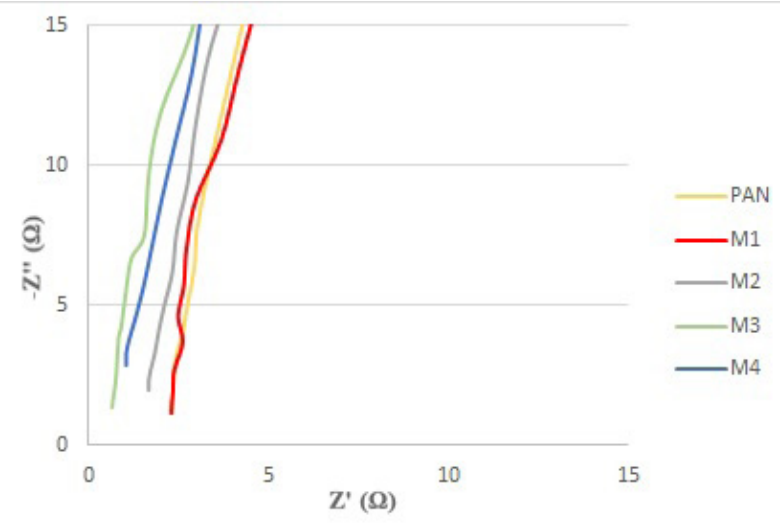

Fig. 6 AC Impedance spectra of separators

increased compared to the neat PAN separator, there is a competitive relationship between these two important operational parameters, which can possibly influence the ion conductivity [54]. As seen in Fig. 5, the ion conductivity of the M3 separator considerably increased from 1.1 to $3.16 \mathrm{mS} / \mathrm{cm}$ for M3 in comparison to the PAN neat separator by enhancing the amounts of PVA hydrophilic polymer and MA cross-linking agent.

\subsection{The analysis of cycling performance and rate capability}

Fig. 7 shows the cycling performance of the neat PAN and M3 separators. As shown in Fig. 7, an excellent lithium-ion battery performance occurred when the M3 separator was used. In addition, the discharge capacity of PAN and M3 separators declined from 151.01 to 135.95 and from 154.61 to $145.53 \mathrm{~mA} . \mathrm{h} / \mathrm{g}$, respectively, after 100 cycles, which may be due to the enhancement of the ion conductivity and appropriate structural expansion [5]. Higher resistances were eventuated in lower ion conductivities, which reduced the discharge capacities. The increased amount of cell resistance could be attributed to the physical changes

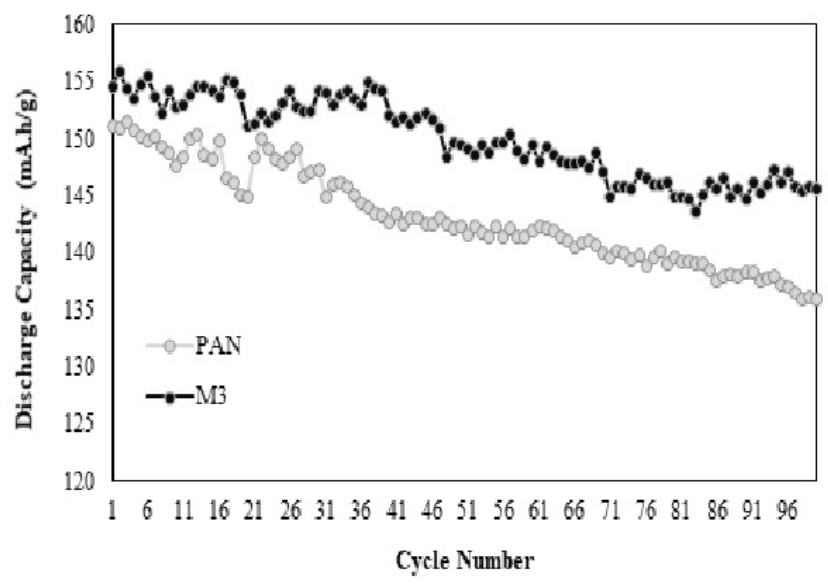

Fig. 7 The cycling performance of PAN and M3 separators in their active materials and interfaces [26]. The cells were enforced to charge and discharge at high rates to ensure the appropriate capacity rate (C-rate) performance of the LiFePO4/Li cells including a separator. A good cycling performance can be due to two reasons. First, the porous structure of the M3 separator results in enhanced liquid electrolyte wettability and ion transport. Second, the higher capacity of the M3 separator to maintain a more liquid electrolyte compared with the neat PAN separator leads prevents leakage in the liquid electrolyte during cycling, and then the separator improved cyclability.

Fig. 8 shows the rate capability of the neat PAN and M3 separator. At $0.1 \mathrm{C}$, the $\mathrm{C}$-rate of the cells including neat PAN separator is $152.81 \mathrm{mAh} / \mathrm{g}$, which decreased to approximately $106.76 \mathrm{mAh} / \mathrm{g}$ when the capacity reaches $2 \mathrm{C}$. However, the C-rate of the cells containing M3 separator is higher than PAN and equals to 155.71 at $0.1 \mathrm{C}$ and $129.64 \mathrm{mAh} / \mathrm{g}$ at $2 \mathrm{C}$, respectively. It is worth mentioning that ion conductivity and the interfacial resistance of the separators are considered as two prominent parameters, which significantly influence the C-rate efficiency [3]. The superior ion conductivity as well as the more appropriate interfacial properties of the cells including PAN/PVA/ MA after uptaking liquid electrolyte enhanced the C-rate of the M3 separator in comparison to the PAN separator.

\section{Conclusion}

In this study, PAN membranes were fabricated by applying the electrospinning technique for using as a separator in lithium-ion batteries. MA and PVA in the separators were used as hydrophilic and crosslinking materials, respectively. In addition, the optimum ratio of MA to PVA was determined to introduce the best mechanical, morphological, and electrochemical performance of PAN/PVA/MA

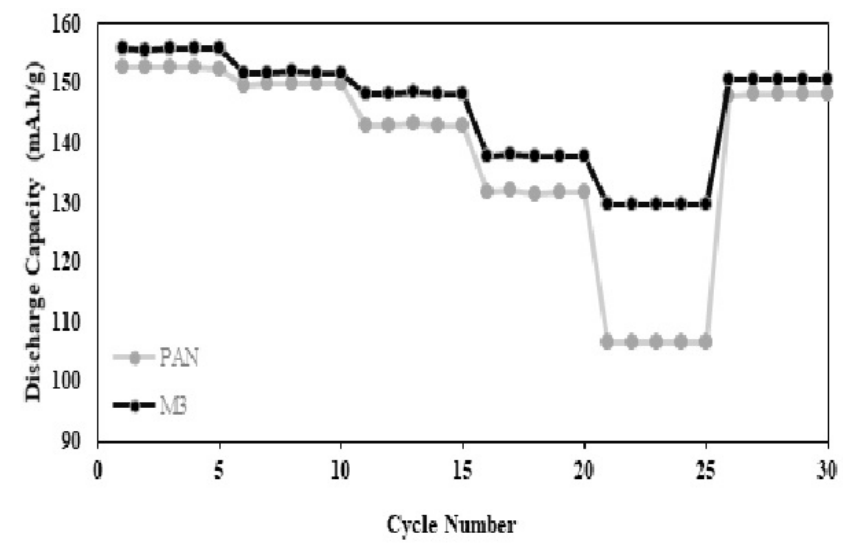

Fig. 8 The results of C-rate capability tests for the cells containing PAN and M3 
electrospun separators inside the lithium-ion battery. The results showed that PAN separator in the presence of PVA and MA with the ratio of $37.5 \%$ (37.5\% MA / PVA+MA), known as M3 in the present study, had the best performance in comparison to other fabricated separators. Additionally, the electrolyte uptake and the thermal shrinkage of M3 separator were calculated as $1172 \%$ and $7.4 \%$, respectively, while these values were reported as $490.5 \%$ and $14.5 \%$ for neat PAN in the same experimental condition.

\section{References}

[1] Marzouk, S. A. M., Al-Marzouqi, M. H., Abdullatif, N., Ismail, Z. M. "Removal of percentile level of H2S from pressurized H2S$\mathrm{CH} 4$ gas mixture using hollow fiber membrane contactors and absorption solvents", Journal of Membrane Science, 360(1-2), pp. 436-441, 2010.

https://doi.org/10.1016/j.memsci.2010.05.046

[2] Masudy-Panah, S., Katal, R., Khiavi, N. D., Shekarian, E., Hu, J., Gong, X. "A high-performance cupric oxide photocatalyst with palladium light trapping nanostructures and a hole transporting layer for photoelectrochemical hydrogen evolution", Journal of Materials Chemistry A, 7(39), pp. 22332-22345, 2019. https://doi.org/10.1039/C9TA06771J

[3] Shekarian, E., Jafari Nasr, M. R., Mohammadi, T., Bakhtiari, O., Javanbakht, M. "Preparation of 4A zeolite coated polypropylene membrane for lithium-ion batteries separator", Journal of Applied Polymer Science, 136(32), Article Number: 47841, 2019. https://oi.org/10.1002/app.47841

[4] Zhang, S. S. "A review on the separators of liquid electrolyte Li-ion batteries", Journal of Power Sources, 164(1), pp. 351-364, 2007. https://doi.org/10.1016/j.jpowsour.2006.10.065

[5] Arora, P., Zhang, Z. "Battery Separators", Chemical Reviews, 104(10), pp. 4419-4462, 2004. https://doi.org/10.1021/cr020738u

[6] Koombhongse, S., Liu, W., Reneker, D. H. "Flat polymer ribbons and other shapes by electrospinning", Journal of Polymer Science Part B: Polymer Physics, 39(21), pp. 2598-2606, 2001. https://doi.org/10.1002/polb.10015

[7] Choi, S. S., Chu, B. Y., Hwang, D. S., Lee, S. G., Park, W. H., Park, J. K. "Preparation and characterization of polyaniline nanofiber webs by template reaction with electrospun silica nanofibers", Thin Solid Films, 477(1-2), pp. 233-239, 2005. https://doi.org/10.1016/j.tsf.2004.08.143

[8] Raghavan, P., Lim, D. H., Ahn, J. H., Nah, C., Sherrington, D. C., Ryu, H. S., Ahn, H. J. "Electrospun polymer nanofibers: The booming cutting edge technology", Reactive and Functional Polymers, 72(12), pp. 915-930, 2012. https://doi.org/10.1016/j.reactfunctpolym.2012.08.018

[9] Huai, Y., Gao, J., Deng, Z., Suo, J. "Preparation and characterization of a special structural poly(acrylonitrile)-based microporous membrane for lithium-ion batteries", Ionics, 16(7), pp. 603-611, 2010 .

https://doi.org/10.1007/s11581-010-0431-4
The comparison indicated the superior performance of the M3 modified separator compared with the neat PAN. Based on the results of the ion conductivity test, this separator indicated a value of $3.16 \mathrm{mS} / \mathrm{cm}$ which was better than the other separators.

\section{Acknowledgement}

We would like to thank Dr. Reza Danesh Talab for his valuable help in this study.

[10] Zhu, Y., Yin, M., Liu, H., Na, B., Lv, R., Wang, B., Huang, Y. "Modification and characterization of electrospun poly (vinylidene fluoride)/poly (acrylonitrile) blend separator membranes", Composites Part B: Engineering, 112, pp. 31-37, 2017. https://doi.org/10.1016/j.compositesb.2016.12.025

[11] Agubra, V. A., De la Garza, D., Gallegos, L., Alcoutlabi, M. "ForceSpinning of polyacrylonitrile for mass production of lithium-ion battery separators", Journal of Applied Polymer Science, 133(1), Article Number: 42847, 2016.

https://doi.org/10.1002/app.42847

[12] He, C., Liu, J., Li, J., Zhu, F., Zhao, H. "Blending based polyacrylonitrile/poly (vinyl alcohol) membrane for rechargeable lithium ion batteries", Journal of Membrane Science, 560, pp. 30-37, 2018. https://doi.org/10.1016/j.memsci.2018.05.013

[13] Gaaz, T. S., Hussein, E. K., Al-Amiery, A. "Physical Properties of Halloysite Nanotubes-Polyvinyl Alcohol Nanocomposites Using Malonic Acid Crosslinked", Jurnal Kejuruteraan, 29(2), pp. 71-77, 2017.

https://doi.org/10.17576/jkukm-2017-29(2)-02

[14] Zhang, Y., Zhu, P. C., Edgren, D. "Crosslinking reaction of poly(vinyl alcohol) with glyoxal", Journal of Polymer Research, 17(5), pp. 725-730, 2010 .

https://doi.org/10.1007/s10965-009-9362-z

[15] Yeom, C. K., Lee, K. H. "Pervaporation separation of water-acetic acid mixtures through poly(vinyl alcohol) membranes crosslinked with glutaraldehyde", Journal of Membrane Science, 109(2), pp. 257-265, 1996.

https://doi.org/10.1016/0376-7388(95)00196-4

[16] Jian, S., Ming, S. X. "Crosslinked PVA-PS thin-film composite membrane for reverse osmosis", Desalination, 62, pp. 395-403, 1987. https://doi.org/10.1016/0011-9164(87)87040-6

[17] Majumdar, S., Adhikari, B. "Polyvinyl alcohol: A taste sensing material", Sensors and Actuators B: Chemical, 114(2), pp. 747-755, 2006.

https://doi.org/10.1016/j.snb.2005.07.052

[18] Ivanishchev, A. V., Bobrikov, I. A., Ivanishcheva, I. A., Ivanshina, O. Y. "Study of structural and electrochemical characteristics of LiNi0.33Mn0.33Co0.33O2 electrode at lithium content variation", Journal of Electroanalytical Chemistry, 821, pp. 140-151, 2018. https://doi.org/10.1016/j.jelechem.2018.01.020 
[19] Makhov, S. V., Ivanishchev, A. V., Ushakov, A. V., Makhov, D. V. "Electrospun Separation Material for Lithium-Ion Batteries: Synthesis and Study of Physical and Electrochemical Properties", Energies, 13(1), Article Number: 18, 2020. https://doi.org/10.3390/en13010018

[20] Ivanishchev A. V., Churikov, A. V., Ivanishcheva, I. A., Ushakov, A. V., Sneha, M. J., Babbar, P., Dixit, A. "Models of lithium transport as applied to determination of diffusion characteristics of intercalation electrodes", Russian Journal of Electrochemistry, 53(7), pp. 706-712, 2017. https://doi.org/10.1134/S1023193517070047

[21] Makhov, S. V., Ivanishchev, A. V. "Long-Term Cycling Behavior of Electrospun Separators for Lithium-Ion Batteries: A Comparison with Conventional Separators", Energies, 13(9), Article Number: 2183, 2020. https://doi.org/10.3390/en13092183

[22] Ivanishchev, A. V., Gridina, N. A., Rybakov, K. S., Ivanishcheva, I. A., Dixit, A. "Structural and electrochemical investigation of lithium ions insertion processes in polyanionic compounds of lithium and transition metals", Journal of Electroanalytical Chemistry, 860, Article Number: 113894, 2020. https://doi.org/10.1016/j.jelechem.2020.113894

[23] Bolto, B., Tran, T., Hoang, M., Xie, Z. "Crosslinked poly(vinyl alcohol) membranes", Progress in Polymer Science, 34(9), pp. 969-981, 2009.

https://doi.org/10.1016/j.progpolymsci.2009.05.003

[24] Qiu, K., Netravali, A. N. "Halloysite nanotube reinforced biodegradable nanocomposites using noncrosslinked and malonic acid crosslinked polyvinyl alcohol", Polymer Composites, 34(5), pp. 799-809, 2013.

https://doi.org/10.1002/pc.22482

[25] Stevens, E. S. "Green Plastics: An Introduction to the New Science of Biodegradable Plastics", Princeton University Press, Princeton, NJ, USA, 2002.

[26] Shekarian, E., Jafari Nasr, M. R., Mohammadi, T., Bakhtiari, O., Javanbakht, M. "Enhanced wettability and electrolyte uptake of coated commercial polypropylene separators with inorganic nanopowders for application in lithium-ion battery", Journal of Nanostructures, 9(4), pp. 736-750, 2019.

https://doi.org/10.22052/JNS.2019.04.015

[27] Hakkak, F., Salehi, M. M. "Preparation of Porous Polyacrylonitrile/ Poly (vinylidene fluoride) Nanofibers via Selective Dissolution of Electrospun PAN/PVdF/PMMA Nanofibers", European Journal of Advances in Engineering and Technology, 2(4), pp. 8-10, 2015.

[28] Nakhjiri, A. T., Heydarinasab, A., Bakhtiari, O., Mohammadi, T. "The effect of membrane pores wettability on $\mathrm{CO} 2$ removal from $\mathrm{CO} 2 / \mathrm{CH} 4$ gaseous mixture using $\mathrm{NaOH}, \mathrm{MEA}$ and TEA liquid absorbents in hollow fiber membrane contactor", Chinese Journal of Chemical Engineering, 26(9), pp. 1845-1861, 2018. https://doi.org/10.1016/j.cjche.2017.12.012

[29] Stalin, S., Choudhury, S., Zhang, K., Archer, L. A. "Multifunctional Cross-Linked Polymeric Membranes for Safe, High-Performance Lithium Batteries", Chemistry of Materials, 30(6), pp. 2058-2066, 2018.

https://doi.org/10.1021/acs.chemmater.7b05353
[30] Yu, L., Miao, J., Jin, J., Lin, J. Y. S. "A comparative study on polypropylene separators coated with different inorganic materials for lithium-ion batteries", Frontiers of Chemical Science and Engineering, 11(3), pp. 346-352, 2017. https://doi.org/10.1007/s11705-017-1648-9

[31] Kim, S. J., Park, S. J., Kim, S. I. "Swelling behavior of interpenetrating polymer network hydrogels composed of poly(vinyl alcohol) and chitosan", Reactive and Functional Polymers, 55(1), pp. $53-59,2003$ https://doi.org/10.1016/S1381-5148(02)00214-6

[32] Mansur, H. S., Sadahira, C. M., Souza, A. N., Mansur, A. A. P. "FTIR spectroscopy characterization of poly (vinyl alcohol) hydrogel with different hydrolysis degree and chemically crosslinked with glutaraldehyde", Materials Science and Engineering: C, 28(4), pp. 539-548, 2008. https://doi.org/10.1016/j.msec.2007.10.088

[33] Yalcinkaya, F., Yalcinkaya, B., Pazourek, A., Mullerova, J., Stuchlik, M., Maryska, J. "Surface Modification of Electrospun PVDF/PAN Nanofibrous Layers by Low Vacuum Plasma Treatment", International Journal of Polymer Science, 2016, Article ID: 4671658, 2016. https://doi.org/10.1155/2016/4671658

[34] Gohil, J. M., Bhattacharya, A., Ray, P. "Studies On The Crosslinking Of Poly (Vinyl Alcohol)", Journal of Polymer Research, 13(2), pp. 161-169, 2006 https://doi.org/10.1007/s10965-005-9023-9

[35] Upadhyay, D., Bhat, N. V. "Pervaporation studies of gaseous plasma treated PVA membrane", Journal of membrane science, 239(2), pp. 255-263, 2004. https://doi.org/10.1016/j.memsci.2004.03.041

[36] Arifeen, W. U., Kim, M., Ting, D., Kurniawan, R., Choi, J., Yoo, K., Ko, T. J. "Hybrid thermal resistant electrospun polymer membrane as the separator of lithium ion batteries", Materials Chemistry and Physics, 245, Article Number: 122780, 2020. https://doi.org/10.1016/j.matchemphys.2020.122780

[37] Park, B., Hong, J. H., Kim, H. "Spinline behavior and web morphology in multi-nozzle electrospinning of PAN/DMF solution", Fibers and Polymers, 13(7), pp. 850-854, 2012. https://doi.org/10.1007/s12221-012-0850-0

[38] Zainab, G., Wang, X., Yu, J., Zhai, Y., Babar, A. A., Xiao, K., Ding, B. "Electrospun polyacrylonitrile/polyurethane composite nanofibrous separator with electrochemical performance for high power lithium ion batteries", Materials Chemistry and Physics, 182, pp. 308-314, 2016 https://doi.org/10.1016/j.matchemphys.2016.07.037

[39] Nakhjiri, A. T., Heydarinasabi, A. "Modeling and numerical simulation of two species separation using non-linear HPLC chromatography", Applied Research Journal, 3(9), pp. 266-270, 2017.

[40] Haider, A., Haider, S., Kang, I. K. "A comprehensive review summarizing the effect of electrospinning parameters and potential applications of nanofibers in biomedical and biotechnology", Arabian Journal of Chemistry, 11(8), pp. 1165-1188, 2018. https://doi.org/10.1016/j.arabjc.2015.11.015 
[41] Cheng, Z., Zhang, Y., Han, Z., Cui, L., Kang, L., Zhang, F. "A novel preparation of anti-layered poly(vinylalcohol)-polyacrylonitrile (PVA/PAN) membrane for air filtration by electrospinning", RSC Advances, 6(88), pp. 85545-85550, 2016.

https://oi.org/10.1039/C6RA13971J

[42] Jung, Y. C., Kim, S. K., Kim, M. S., Lee, J. H., Han, M. S., Kim, D. H., Shin, W. C., Ue, M., Kim, D. W. "Ceramic separators based on $\mathrm{Li}+$-conducting inorganic electrolyte for high-performance lithium-ion batteries with enhanced safety", Journal of Power Sources, 293, pp. 675-683, 2015.

https://doi.org/10.1016/j.jpowsour.2015.06.001

[43] Lee, Y., Lee, H., Lee, T., Ryou, M. H., Lee, Y. M. "Synergistic thermal stabilization of ceramic/co-polyimide coated polypropylene separators for lithium-ion batteries", Journal of Power Sources, 294, pp. 537-544, 2015.

https://doi.org/10.1016/j.jpowsour.2015.06.106

[44] Zhu, G., Wang, F., Xu, K., Gao, Q., Liu, Y. "Study on Properties of Poly(vinyl alcohol)/Polyacrylonitrile Blend Film", Polímeros: Ciência e Tecnologia, 23(2), pp. 146-151, 2013. https://doi.org/10.4322/polimeros.2013.076

[45] Dastidar, T. G., Netravali, A. N. "Green'crosslinking of native starches with malonic acid and their properties", Carbohydrate Polymers, 90(4), pp. 1620-1628, 2012. https://doi.org/10.1016/j.carbpol.2012.07.041

[46] Chatterjee, U., Jewrajka, S. K., Guha, S. "Dispersion of functionalized silver nanoparticles in polymer matrices: Stability, characterization, and physical properties", Polymer Composites, 30(6), pp. 827-834, 2009.

https://doi.org/10.1002/pc.20655

[47] Nakhjiri, A. T., Heydarinasab, A., Bakhtiari, O., Mohammadi, T. "Influence of non-wetting, partial wetting and complete wetting modes of operation on hydrogen sulfide removal utilizing monoethanolamine absorbent in hollow fiber membrane contactor", Sustainable Environment Research, 28(4), pp. 186-196, 2018. https://doi.org/10.1016/j.serj.2018.02.003
[48] Jeon, H., Jin, S. Y., Park, W. H., Lee, H., Kim, H. T., Ryou, M. H., Lee, Y. M. "Plasma-assisted water-based $\mathrm{A} 12 \mathrm{O} 3$ ceramic coating for polyethylene-based microporous separators for lithium metal secondary batteries", Electrochimica Acta, 212, pp. 649-656, 2016. https://doi.org/10.1016/j.electacta.2016.06.172

[49] Li, N., Xiao, C., An, S., Hu, X. "Preparation and properties of PVDF/PVA hollow fiber membranes", Desalination, 250(2), pp. 530-537, 2010. https://doi.org/10.1016/j.desal.2008.10.027

[50] Patnaik, A., Rengasamy, R. S., Kothari, V. K., Ghosh, A. "Wetting and Wicking in Fibrous Materials", Textile Progress, 38(1), pp. 1-105, 2006. https://doi.org/10.1533/jotp.2006.38.1.1

[51] Bhushan, B., Jung, Y. C., Koch, K. "Micro-, nano-and hierarchical structures for superhydrophobicity, self-cleaning and low adhesion", Philosophical Transactions of the Royal Society A: Mathematical, Physical and Engineering Sciences, 367(1894), pp. 1631-1672, 2009 https://doi.org/10.1098/rsta.2009.0014

[52] Mohamed, A. M. A., Abdullah, A. M., Younan, N. A. "Corrosion behavior of superhydrophobic surfaces: A review", Arabian Journal of Chemistry, 8(6), pp. 749-765, 2015. https://doi.org/10.1016/j.arabjc.2014.03.006

[53] Yanilmaz, M., Chen, C., Zhang, X. "Fabrication and characterization of SiO2/PVDF composite nanofiber-coated PP nonwoven separators for lithium-ion batteries", Journal of Polymer Science Part B: Polymer Physics, 51(23), pp. 1719-1726, 2013. https://doi.org/10.1002/polb.23387

[54] Liu, H., Xu, J., Guo, B., He, X. "Effect of $\mathrm{SiO} 2$ content on performance of polypropylene separator for lithium-ion batteries", Journal of Applied Polymer Science, 131(23), Article Number: 41156, 2014. https://doi.org/10.1002/app.41156

[55] Zhang, Z., Yuan, W., Li, L. "Enhanced wettability and thermal stability of nano-SiO2/poly(vinyl alcohol)-coated polypropylene composite separators for lithium-ion batteries", Particuology, 37, pp. 91-98, 2018. https://doi.org/10.1016/j.partic.2017.10.001 